\title{
Corley-Jacobson dispersion relation and trans-Planckian inflation
}

\author{
Jérôme Martin* \\ Institut d'Astrophysique de Paris, 98bis boulevard Arago, 75014 Paris, France \\ Robert H. Brandenberger ${ }^{\dagger}$ \\ Theory Division, CERN, CH-1211 Geneva 23, Switzerland \\ (Received 24 January 2002; published 9 May 2002)
}

\begin{abstract}
In this paper we study the dependence of the spectrum of fluctuations in inflationary cosmology on possible effects of trans-Planckian physics, using the Corley-Jacobson dispersion relations as an example. We compare the methods used in our previous work with the WKB approximation, give a new exact analytical result, and study the dependence of the spectrum obtained using the approximate method of our previous work on the choice of the matching time between different time intervals. We also comment on recent work subsequent to our previous work on the trans-Planckian problem for inflationary cosmology.
\end{abstract}

DOI: 10.1103/PhysRevD.65.103514

PACS number(s): 98.80.Hw, 98.80.Cq

\section{INTRODUCTION}

The trans-Planckian problem of inflation [1] is the following: in many models of inflation, the phase of accelerated expansion lasts so many $e$-foldings that the comoving lengths corresponding to present day cosmological scales were much smaller than the Planck length at the beginning of inflation. Hence, one may wonder whether the "standard" predictions of inflation, in particular the fact that the power spectrum of cosmological perturbations is close to scale invariant, will be changed if the laws of physics beyond the Planck scale are different from the ones which rule the low energy phenomena. The usual calculations of the spectrum of cosmological perturbations (see e.g. [2] for a comprehensive review) are based on the use of classical general relativity coupled to a weakly interacting scalar field, and on linearizing the resulting equations of motion about a classical background cosmology. The validity of this approach in the transPlanckian regime is highly doubtful.

In the context of inflation, this question was first addressed in Ref. [1]. To calculate the power spectrum of cosmological perturbations, the main equation that needs to be solved is the equation of a parametric oscillator with a timedependent frequency which is a function of the scale factor $a(\eta)$ (and its derivatives) and of the dispersion relation $\omega_{\text {phys }}(k)$, where $k$ indicates the physical wave number related to the comoving wave number $n$ by $k=n / a$. In the usual discussions, the dispersion relation is taken to be linear as appropriate for a free field theory. The method used in Ref. [1] was to replace the linear relation $\omega_{\text {phys }}=k$ by a nonstandard one that mimics possible modifications of the physics in the ultraviolet regime. Based on similar approaches $[3,4]$ used to study the possible dependence of Hawking radiation on trans-Planckian physics, two classes of dispersion relations were considered $\left[n_{\text {eff }} \equiv a(\eta) \omega_{\text {phys }}(n / a)\right]$ :

$$
n_{\mathrm{eff}}=n \frac{\lambda}{l_{\mathrm{C}}} \tanh ^{1 / p}\left[\left(\frac{l_{\mathrm{C}}}{\lambda}\right)^{p}\right]
$$

\footnotetext{
*Electronic address: jmartin@iap.fr

${ }^{\dagger}$ On leave from Department of Physics, Brown University, Providence, RI 02912. Electronic address: rhb@ het.brown.edu
}

$$
n_{\mathrm{eff}} \equiv \sqrt{n^{2}+n^{2} b_{1}\left(\frac{l_{\mathrm{C}}}{\lambda}\right)^{2}},
$$

where $\lambda$ denotes the physical wavelength of a given mode, and $l_{\mathrm{C}}$ is a characteristic length expected to be determined by the Planck scale. The first one is the Unruh dispersion relation [3] whereas the second one is the Corley-Jacobson relation [4] $\left(b_{1}\right.$ is an arbitrary number which can be positive or negative). In fact, in Ref. [1] a generalization of the CorleyJacobson dispersion relation was considered.

In [1], the problem was investigated for the class of scale factors corresponding to power-law inflation, i.e. $a(\eta)$ $=l_{0}|\eta|^{1+\beta}, \beta \leqslant-2$ where $l_{0}$ has the dimension of a length and is equal to the Hubble radius during inflation if $\beta$ $=-2$ (de Sitter inflation). The example of the Unruh dispersion relation was treated only for $\beta=-2$ whereas the second dispersion relation was studied for any value of $\beta \leqslant-2$. It was found that no modifications in the spectrum of fluctuations arise in the first case, whereas some differences can show up in the second case if $b_{1}<0 .{ }^{1}$

The aim of this paper is to return to the example of the Corley-Jacobson dispersion relations, making use of a new exact analytical solution. This one allows us to address some technical points that have been raised recently in the literature and to compare the method of Ref. [1] with the other methods used in the works subsequent to [1]. Explicitly, in Sec. II, we compare the Wigner-Kramers-Brillouin (WKB) approximation method used in Ref. [5] to calculate the power spectrum with the method used in Ref. [1]. In Sec. III, we consider the exact formula mentioned above which allows us to make a smooth transition from the region where the sub-Planckian effects are important to the region where the dispersion relation is standard. We take advantage of the fact that this new solution is also valid for $b_{1}<0$ to discuss

\footnotetext{
${ }^{1}$ This result was obtained assuming that the initial state is the "minimizing energy state." In Ref. [1], another state was also considered, but only to demonstrate that the final spectrum depends on the choice of the initial state. As stressed in Ref. [1], the "minimizing energy state" is the only physically well-motivated state.
} 
in more detail than in Ref. [1] the physical meaning of complex solutions in the sub-Planckian region. In the fourth section, we study the consequences for the matching time used in the approximate analysis of [1] and show that the use of an incorrect matching time will lead to artificial oscillations in the spectrum. ${ }^{2}$ First, however, we comment on some of the recent work on this subject.

Following Ref. [1], there has been a significant amount of work on the trans-Planckian problem for inflationary cosmology. The work has focused on two issues. The first is how broad the class of dispersion relations is for which there is a change in the spectrum of fluctuations, and whether there are other features in the linear perturbations which can be used to probe trans-Planckian physics. The second is whether the back reaction of the excess fluctuations produced by modified dispersion relations is important and can have interesting consequences.

Let us for the moment focus on the issue of the class of dispersion relations for which a modification of the spectrum of fluctuations is found. In [6], the case of Unruh dispersion relations was studied in a de Sitter Universe, and no change in the spectrum was found, in agreement with the corresponding results in [1]. No dispersion relations of CorleyJacobson type were studied in [6]. ${ }^{3}$ In $[5,7]$ the transPlanckian problem of inflationary cosmology was addressed assuming that the mode wave function is of WKB-type form. Once again, no changes in the spectrum were found. It has been pointed out in [8] that for dispersion relations which lead to adiabatic evolution of the states on sub-Planckian scales - and WKB states fall into this category-there are no changes in the spectrum compared to what is obtained with a linear dispersion relation. As shown in [8], the evolution in the case of the Corley-Jacobson dispersion relations with a negative sign of $b_{1}$ is not adiabatic, and this is the reason that the spectrum is modified. Note that the change in the spectrum of fluctuations in the case of Corley-Jacobson dispersion relations with $b_{1}<0$ was confirmed in [7], where these dispersion relations were labeled as "exceptional forms of $\omega(k) . "$ Other dispersion relations were studied in [9-12].

The question of a possible dependence of the predictions for linear cosmological fluctuations on trans-Planckian physics was recently considered in the context of possible ap-

\footnotetext{
${ }^{2}$ As pointed out in [5], there was a mistake in Sec. V B 2 of the first version of [1]: the incorrect choice of the matching time in the Corley-Jacobson case with $b_{1}>0$ led to a spectrum which was the usual one times a complicated oscillatory function, instead of to an unmodified spectrum.

${ }^{3}$ On this basis, the claims of Ref. [5] that "contradictory results" or "opposite results" were found in Refs. [1] and [6] are misleading. Indeed, the only common case between these two articles is the case of the Unruh dispersion relation in de Sitter spacetime for which exactly the same conclusion was obtained in Refs. [1] and [6].

${ }^{4}$ The goal of [10] was to provide a new model for dark energy (see, however, [13]), and in [11] and [12] novel dispersion relations were used to yield realizations of the varying speed of light scenario [11] and to obtain inflation from radiation [12].
}

proaches to quantum gravity in $[14-17]$. In $[14,16,17]$ the starting point was modifications of the commutation relations stemming from general considerations of short-distance quantum gravitational effects, and in [15] consequences of short-distance non-commutative geometry were investigated. Interesting deviations of the spectrum of fluctuations from the usual results were found. Specifically, the analysis of [15] showed that non-Gaussian fluctuations are expected, and the analysis of [17] revealed changes to the spectral shape.

To summarize the current state of knowledge on the issue of the possible dependence of the spectrum of fluctuations on trans-Planckian physics, if the dispersion relation leads to adiabatic evolution of the vacuum state on sub-Planckian length scales, then the spectrum is not modified [8,5,7]. However, this restriction on the class of dispersion relations may exclude the cases of actual physical interest $[15,17]$. String theory, M-theory, non-commutative geometry and discrete quantum gravity can all lead to much more drastic changes in the effective dispersion relation and may hence result in changes in the spectrum of fluctuations which can be probed observationally with current and future cosmic microwave background (CMB) experiments. Recently, two concrete examples where a change in the spectrum is obtained were studied in Refs. [13,18].

The second issue raised in recent work, in particular in $[19,7]$ is whether, if the power spectrum is indeed modified by trans-Planckian physics, there is a back reaction problem. ${ }^{5}$ The back reaction problem is the following: when viewed at late times, the modified dispersion relations studied in [1] and in subsequent papers lead to mode functions which during inflation on length scales larger than $l_{\mathrm{C}}$ but smaller than the Hubble radius are excited compared to the adiabatic vacuum. These fluctuations carry energy and momentum, and this energy could be so large as to turn off inflation, in a similar manner as the back reaction of cosmological fluctuations in models of chaotic inflation [20] can build up and terminate inflation. This is a very interesting issue which merits detailed study.

\section{WKB APPROXIMATION VERSUS PIECEWISE SOLUTIONS}

The equation of motion that needs to be solved in order to compute the power spectrum of cosmological perturbations is

$$
\mu^{\prime \prime}+\omega^{2}(n, \eta) \mu=0
$$

where the expression for $\omega$ is given below [see Eq. (7)]. Usually, Eq. (3) possesses two regimes depending on

\footnotetext{
${ }^{5}$ Clearly, the back reaction problem can only be raised as a second question once it has been established that there are important modifications to the fluctuation spectrum.
} 
whether the wavelength $\lambda$ of the mode is smaller or larger than the Hubble radius $l_{\mathrm{H}}$. In the present context, we deal with three different regimes. The first one is when $\lambda \ll l_{\mathrm{C}}$ $\ll l_{\mathrm{H}}$. This defines region I of Ref. [1]. The second regime is such that $l_{\mathrm{C}} \ll \lambda \ll l_{\mathrm{H}}$ and corresponds to region II of Ref. [1]. Finally, the third regime is when $l_{\mathrm{C}} \ll l_{\mathrm{H}} \ll \lambda$; this is region III of Ref. [1]. We recover the usual result for the spectrum of fluctuations if in region II $\mu$ is given by a single branch with a coefficient proportional to $1 / \sqrt{n}$ multiplying the plane wave solution.

The method used in Ref. [1] to determine the solution of Eq. (3) is to find solutions in the three regions and to match them at the boundaries between the regions. The method used in Ref. [5] is different and consists in utilizing the WKB approximation. In that case, the solution of Eq. (3) is given by

$$
\mu_{\mathrm{WKB}}=\frac{1}{\sqrt{2 \omega(\eta)}} \exp \left[ \pm i \int_{\eta_{\mathrm{i}}}^{\eta} \omega(\tau) \mathrm{d} \tau\right],
$$

where $\eta_{\mathrm{i}}$ is some initial time.

In cases where the adiabatic approximation is justified, the two methods are equivalent. To verify this, consider first Unruh's dispersion relation. Inserting the dispersion relation into Eq. (4) (using the minus sign) yields in region I

$$
\mu_{\mathrm{WKB}}=\frac{1}{2} \sqrt{\frac{\epsilon}{\pi}}\left|\eta_{\mathrm{i}}\right|^{1 / 2}\left(\left|\frac{\eta}{\eta_{\mathrm{i}}}\right|\right)^{-2 \pi i / \epsilon+1 / 2} .
$$

This is nothing but Eq. (66) of Ref. [1] [i.e. Eq. (54) in the limit when $\epsilon \equiv l_{\mathrm{C}} / l_{0}$ is small], properly normalized according to Eqs. (56) and (57) of that reference. Similarly, in the case of the Corley-Jacobson dispersion relation (with $b_{1}>0$, in which case the adiabatic condition is satisfied [5]), Eq. (4) becomes

$$
\begin{aligned}
\mu_{\mathrm{WKB}}= & 2^{-1 / 2}\left(\frac{n \epsilon}{2 \pi}\right)^{-1 / 2} n^{-1 / 2} b_{1}^{-1 / 4}|\eta|^{-1 / 2} \\
& \times \exp \left[\mp i b_{1}^{1 / 2}\left(\frac{n \epsilon}{4 \pi}\right) n\left(|\eta|^{2}-\left|\eta_{\mathrm{i}}\right|^{2}\right)\right] .
\end{aligned}
$$

Again, this is the same result as obtained with the matching technique in Ref. [1]. To see this, take Eq. (118) of that reference, which provides the solution in region I, consider the limit when $z$ is large and use Eqs. (126) and (127) with the lower sign. In region II, the solution is simply given by plane waves $\mu \simeq B_{1} e^{i n \eta}+B_{2} e^{-i n \eta}$ in agreement with Eq. (14) of Ref. [1].

The advantage of the WKB method over the method used in Ref. [1] is that one does not have to perform the matching between the different regions. The disadvantage is that the WKB method applies only to examples in which the adiabaticity condition is satisfied. In the next section, we consider an exact model which allows us to avoid the matching between region I and II. In the third section, we study the consequences for the matching time and show that an incor- rect matching time could lead to artificial oscillations in the spectrum, as pointed out in Ref. [5].

\section{THE EXACT MODEL}

The effective time dependent frequency is given by the equation

$$
\omega^{2}(n, \eta)=n_{\mathrm{eff}}^{2}-\frac{a^{\prime \prime}}{a} .
$$

We consider the Corley-Jacobson dispersion relation given in Eq. (1) where, as already mentioned above, $b_{1}$ is an arbitrary number which can be positive or negative. We restrict our consideration to the prototypical model of inflation, i.e. de Sitter inflation. Then the scale factor can be written as $a(\eta)=l_{0} /|\eta|$. If we consider the evolution of the mode well inside horizon, the term $a^{\prime \prime} / a$ can be neglected in Eq. (3). Then, this equation takes on the form

$$
\mu^{\prime \prime}+\left[n^{2}+b_{1}\left(\frac{n^{2} \epsilon}{2 \pi}\right)^{2}|\eta|^{2}\right] \mu=0
$$

where $\epsilon \equiv l_{\mathrm{C}} / l_{0}$. Typically, $\epsilon$ is a very small number of the order $10^{-5}$. We now need to distinguish between the cases of positive or negative $b_{1}$. Let us first concentrate on the $b_{1}$ $>0$ case. We make the following change of variables $-\eta$ $=C x \quad(x>0)$, where $C$ is a constant given by $C$ $=\left[\left(b_{1}\right)^{-1 / 4} / n\right] \sqrt{\pi / \epsilon}$. Then the equation of motion takes the form

$$
\frac{\mathrm{d}^{2} \mu}{\mathrm{d} x^{2}}+\left(\frac{x^{2}}{4}-a\right) \mu=0, \quad a \equiv-\frac{\pi}{\epsilon \sqrt{b_{1}}}<0 .
$$

Clearly, this equation possesses two regimes. The first one, corresponding to region I in Ref. [1], is when the quartic term of the dispersion relation dominates. Expressed in terms of the new variable $x$, this corresponds to $x^{2} / 4 \gg|a|$. On the other hand, region II of Ref. [1] corresponds to a region where the dispersion relation has become standard and $x^{2} / 4$ $\ll|a|$. The general solution of Eq. (9) (valid in both regions) is given in terms of parabolic cylinder functions $E(a, x)$ (see Ref. [21]) and reads

$$
\mu=A_{1} E(a, x)+A_{2} E^{*}(a, x),
$$

where the two constants $A_{1}$ and $A_{2}$ are fixed by the initial conditions when $\eta \rightarrow-\infty$ (i.e. $x \rightarrow \infty$ ). In this limit, the asymptotic behavior is given by [21] 


$$
\lim _{\eta \rightarrow-\infty} \mu=b_{1}^{-1 / 8}\left(\frac{\pi}{\epsilon}\right)^{-1 / 4}\left(\frac{n \epsilon}{2 \pi}\right)^{-1 / 2}|\eta|^{-1 / 2}\left\{A_{1} \exp \left[i b_{1}^{1 / 2} \frac{n^{2} \epsilon}{4 \pi}|\eta|^{2}+i \frac{\pi}{4}+i \frac{\phi_{2}}{2}\right]+A_{2} \exp \left[-i b_{1}^{1 / 2} \frac{n^{2} \epsilon}{4 \pi}|\eta|^{2}-i \frac{\pi}{4}-i \frac{\phi_{2}}{2}\right]\right\}
$$

where $\phi_{2} \equiv \arg \Gamma(1 / 2+i a)$. Thus we reproduce the correct WKB behavior if we take

$$
A_{1}=2^{-1 / 2} \frac{b_{1}^{-1 / 8}}{\sqrt{n}}\left(\frac{\pi}{\epsilon}\right)^{1 / 4} \exp \left[-i\left(b_{1}\right)^{1 / 2}\left(\frac{n^{2} \epsilon}{4 \pi}\right)\left|\eta_{\mathrm{i}}\right|^{2}-i \frac{\pi}{4}-i \frac{\phi_{2}}{2}\right], \quad A_{2}=0 .
$$

Let us now study how this solution behaves in region II. Usually, the solution in this region is given by $1 / \sqrt{2 n} e^{ \pm i n|\eta|}$. It is therefore sufficient to have only one branch proportional to $1 / \sqrt{n}$ to recover the standard scale-invariant spectrum. Using the asymptotic behavior of the parabolic cylinder functions when $x^{2} / 4 \ll|a|$, one finds

$$
\mu \simeq A_{1}\left(\frac{\pi}{\epsilon}\right)^{-1 / 4} b_{1}^{1 / 8} \exp \left[i n|\eta|+i \frac{\pi}{4}\right]
$$

i.e. we precisely recover the conditions necessary to obtain a Harrison-Zeldovich spectrum, due to the fact that $A_{1} \propto 1 / \sqrt{n}$, see Eq. (12).

Let us now turn to the case $b_{1}<0$. An immediate consequence is that the dispersion relation $n_{\text {eff }}(n, \eta)$ vanishes at some point and then becomes complex. Therefore, we face the following problems. First, in the region where $n_{\text {eff }}(n, \eta)$ is small, the term $a^{\prime \prime} / a$ is no longer negligible. In principle it should be taken into account in the equation of motion but no exact solution can then be found. However, this problem is not too serious because the effect of this term on the final spectrum is expected to be small. Second, a much more serious question is the fact that there is a region where we have to quantize a field in the presence of imaginary frequency modes. Although imaginary frequencies are standard in classical physics and in quantum mechanics, they seem to be problematic in the context of quantum field theory [22] although there exist concrete physical situations where they are important [23]. Third, we have to fix the initial conditions in the complex region. A possible choice for the initial conditions, which is consistent with the WKB result, is to keep only the decreasing exponential in the region where the effective frequency becomes complex as proposed in Ref. [1].
Then, an exact solution in terms of parabolic cylinder functions $U(a, x)$ and $V(a, x)$ (see Ref. [21]) can be found. This gives a Harrison-Zeldovich spectrum corrected by oscillations and by an exponential term of the form $e^{A n^{2}}$. This result is in agreement with what was obtained in Ref. [1]. However, this result rests clearly on "non-standard physics" and for this reason is not so attractive. Therefore, it is important to recall that there now exist two cases where the final spectrum is modified and everything is well-defined $[13,18]$.

\section{CONSEQUENCES FOR THE MATCHING PROCEDURE}

We now investigate what we can learn from the previous exact solution with respect to the matching between regions I and II. We concentrate on the Corley-Jacobson case with $b_{1}>0$. A priori, two natural choices for the matching time can be envisaged. The first choice is to match the solutions when $\lambda=l_{\mathrm{C}}$. This amounts to choosing

$$
\left|\eta_{\mathrm{j}}\right|=\left|\eta_{1}\right|=\left(\frac{n l_{\mathrm{C}}}{2 \pi l_{0}}\right)^{-1}
$$

Another possibility is to choose the matching time such that the usual and the extra contribution in the dispersion relation are equal

$$
n^{2} b_{1}\left(\frac{l_{\mathrm{C}}}{\lambda}\right)^{2}=n^{2} \Rightarrow\left|\eta_{\mathrm{j}}\right|=\left|\eta_{1}^{\prime}\right|=\left(\frac{n l_{\mathrm{C}}}{2 \pi l_{0}}\right)^{-1} b_{1}^{-1 / 2} .
$$

This is in fact equivalent to matching the frequencies $n_{\text {eff }}(n, \eta)$. Therefore, $\eta_{1}$ and $\eta_{1}^{\prime}$ are not equal unless $b_{1}=1$. Let us now perform the matching between regions I and II. Then, the coefficient $B_{1}$ is given by:

$$
\begin{aligned}
2 i n B_{1} e^{i n \eta_{\mathrm{j}}=} & 2^{-1 / 2}\left(\frac{n \epsilon}{2 \pi}\right)^{-1 / 2} n^{-1 / 2} b_{1}^{-1 / 4}\left|\eta_{\mathrm{j}}\right|^{-1 / 2} \exp \left[\mp i b_{1}^{1 / 2}\left(\frac{n \epsilon}{4 \pi \beta}\right) n\left(\left|\eta_{\mathrm{j}}\right|^{2}-\left|\eta_{\mathrm{i}}\right|^{2}\right)\right]\left\{i n-\frac{1}{2}(1+\beta)\left|\eta_{\mathrm{j}}\right|^{-1}\right. \\
& \left. \pm i b_{1}^{1 / 2}\left(\frac{n \epsilon}{2 \pi}\right) n\left|\eta_{\mathrm{j}}\right|\right\}
\end{aligned}
$$

For the curly bracket of the above expression one finds

$$
\{\cdots\}_{1}=-\frac{1}{2}(1+\beta)\left|\eta_{\mathrm{j}}\right|^{-1}+i n\left(1 \pm\left|\frac{\eta_{\mathrm{j}}}{\eta_{1}^{\prime}}\right|\right) .
$$


In the same manner, one can determine $B_{2}$ to be

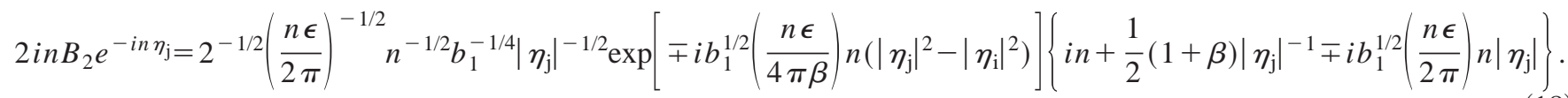

Again, we can write the curly bracket in the previous expression as

$$
\{\cdots\}_{2}=+\frac{1}{2}(1+\beta)\left|\eta_{\mathrm{j}}\right|^{-1}+i n\left(1 \mp\left|\frac{\eta_{\mathrm{j}}}{\eta_{1}^{\prime}}\right|\right) \text {. }
$$

The situation is now clear. If the joining is performed at $\eta_{\mathrm{j}}$ $=\eta_{1}$, the terms proportional to $i n$ in the curly brackets have no reason to cancel out and they are in fact of order one. The term proportional to $\left|\eta_{j}\right|^{-1}$ is very small and can be neglected. Therefore we reach the conclusion that $B_{1} \simeq B_{2}$ and we have oscillations. However, if we perform the matching at $\eta_{\mathrm{j}}=\eta_{1}^{\prime}$ the situation is drastically different. This time one of the curly brackets vanishes and one of the $B_{i}$ 's becomes of the order $\eta_{1}^{\prime-1} \ll 1$, whereas the other one is of order 1 . In other words, only one branch survives, we have no oscillations and the spectrum of fluctuations is unchanged.

A comparison with the exact solution shows that the correct matching time is $\eta_{1}^{\prime}$. Therefore, there are no oscillations in the spectrum in the case $b_{1}>0$, as pointed out in Ref. [5], and the spectrum is unchanged. On the other hand, for $b_{1}$ $<0$ the spectrum is modified, in agreement with the analysis of Ref. [1].

\section{CONCLUSION}

In this paper, we have investigated the following technical points: (i) it has been shown that the method used in Ref. [1] is equivalent to the WKB approach, (ii) a new solution valid in the case of the Corley-Jacobson dispersion relation has been presented, (iii) in the case where piecewise solutions are used, the matching conditions have been studied. It has been demonstrated that the frequencies rather than the wavelengths should be matched (as could have been guessed from the equation of motion) and that if the latter requirement is utilized then artificial oscillations show up in the spectrum. These results complete the study of Ref. [1].

On more general grounds, the conclusion that follows from the previous considerations is that there is a sensitive dependence of the spectrum of cosmological fluctuations on the assumptions made at the level of sub-Planckian physics. A separate issue is whether these modifications are reasonable from a physical point of view. This question cannot be answered in the absence of a realistic theory of physics beyond the Planck scale. To our knowledge, it is still an open problem to derive a dispersion relation from, for example, string theory (see, however, Refs. [14,24] for some recent progress).

\section{ACKNOWLEDGMENTS}

We are grateful to Steve Corley for stimulating discussions and useful comments. We acknowledge support from BROWN-CNRS University which made possible the visit of J.M. to Brown during which some of the work on this project was done, and we are grateful to Herb Fried for his efforts. J.M. thanks the High Energy Group of Brown University for warm hospitality. The research was supported in part by the U.S. Department of Energy under Contract DE-FG0291ER40688, TASK A.
[1] J. Martin and R.H. Brandenberger, Phys. Rev. D 63, 123501 (2001); R.H. Brandenberger and J. Martin, Mod. Phys. Lett. A 16, 999 (2001).

[2] V.F. Mukhanov, H.A. Feldman, and R.H. Brandenberger, Phys. Rep. 215, 203 (1992).

[3] W. Unruh, Phys. Rev. D 51, 2827 (1995).

[4] S. Corley and T. Jacobson, Phys. Rev. D 54, 1568 (1996).

[5] J.C. Niemeyer and R. Parentani, Phys. Rev. D 64, 101301(R) (2001).

[6] J.C. Niemeyer, Phys. Rev. D 63, 123502 (2001).

[7] A.A. Starobinsky, Pis'ma Zh. Éksp. Teor. Fiz. 73, 415 (2001) [JETP Lett. 73, 371 (2001)].

[8] J. Martin and R.H. Brandenberger, astro-ph/0012031.

[9] J. Kowalski-Glikman, Phys. Lett. B 499, 1 (2001).

[10] L. Mersini, M. Bastero-Gil, and P. Kanti, Phys. Rev. D 64, 043508 (2001).

[11] S. Alexander and J. Magueijo, hep-th/0104093.

[12] S. Alexander, R.H. Brandenberger, and J. Magueijo, hep-th/0108190.
[13] M. Lemoine, M. Lubo, J. Martin, and J. Uzan, Phys. Rev. D 65, 023510 (2002).

[14] A. Kempf, Phys. Rev. D 63, 083514 (2001).

[15] C. Chu, B.R. Greene, and G. Shiu, Mod. Phys. Lett. A 16, 2231 (2001).

[16] A. Kempf and J.C. Niemeyer, Phys. Rev. D 64, 103501 (2001).

[17] R. Easther, B.R. Greene, W.H. Kinney, and G. Shiu, Phys. Rev. D 64, 103502 (2001).

[18] R.H. Brandenberger, S.E. Joras, and J. Martin, hep-th/0112122.

[19] T. Tanaka, astro-ph/0012431.

[20] L.R.W. Abramo, R.H. Brandenberger, and V. Mukhanov, Phys. Rev. D 56, 3248 (1997); V. Mukhanov, L.R.W. Abramo, and R.H. Brandenberger, Phys. Rev. Lett. 78, 1624 (1997).

[21] I.S. Gradshteyn and I.M. Ryzhik, Table of Integrals, Series, and Products, 6th ed. (Academic, New York, 2000).

[22] G. Gang, hep-ph/9603166.

[23] L.I. Schiff, H. Snyder, and J. Weinberg, Phys. Rev. 57, 315 (1940).

[24] G. Amelino-Camelia, Int. J. Mod. Phys. D 11, 35 (2002). 